\title{
Accidentes del verbo en las secciones de artículos de investigación*
}

\author{
Verbal Inflection in Research Article Sections \\ Omar Sabaj, ${ }^{1}$ Miguel Fuentes, ${ }^{2}$ Ken Matsuda ${ }^{3}$ \\ ${ }^{1}$ Universidad de La Serena, Prodicyt, Centro de Investigación, La Serena, Chile. \\ Correo electrónico: omarsabaj@userena.cl \\ ${ }^{2}$ Universidad de La Serena, Prodicyt, Centro de Investigación, La Serena, Chile. \\ Correo electrónico: mfuentes@userena.cl \\ ${ }^{3}$ Universidad de La Serena, Prodicyt, Centro de Investigación, La Serena, Chile. \\ Correo electrónico:kmatsuda@userena.cl
}

\begin{abstract}
En este artículo, se describe la variación de los accidentes del verbo en las secciones de artículos de investigación en español. Para ello, se analizaron los accidentes del verbo de 42.086 formas verbales en un corpus representativo de 162 artículos de investigación, pertenecientes a 6 áreas de la ciencia y obtenidos de la base SciELO-Chile. Los resultados generales muestran que los elementos no marcados son siempre más frecuentes, independientemente del área de la ciencia y de la sección. Además, las áreas de la ciencia no afectan los patrones de uso de los verbos. Por último, al analizar la variación por sección, el único accidente que varía sustancialmente es el tiempo verbal. Los resultados de esta investigación son de utilidad para aquellos interesados en la descripción del discurso científico y en la didáctica de la escritura científica.
\end{abstract}

Palabras clave: verbos, artículos de investigación, discurso científico, lingüística de corpus.

This article attempts to describe verbal inflection variation in research article sections written in Spanish. In order to depict these inflections, we analyzed a corpus of 42.086 verbal forms from 162 research papers. These papers are from six scientific areas and were obtained from the Chilean SciELO index. General results show that unmarked elements are always more frequent regardless the scientific area or the section. Besides, we can state that verb patterns are not affected by the scientific area under analysis. Finally, when the sections of the papers were analyzed, we could find out that variation takes place only in tense. These results are useful for both scientific discourse description and the didactics of scientific writing.

Key words: verbs, research articles, scientific discourse, corpus linguistics.

Esta investigación es parte del proyecto Fondecyt 11080097: El artículo de investigación a través de las disciplinas: El caso del indexador Scielo-Chile. 


\section{INTRODUCCIÓN}

La descripción del rol de los verbos en el discurso científico es profusa. En los textos destinados a la difusión del conocimiento, esta categoría gramatical cumple funciones variadas. Entre otras funciones, los verbos se utilizan para posicionar el trabajo propio y para revisar el trabajo de otros, bajo formas de citación, atribución y mención, las cuales varían en las distintas culturas disciplinares. Algunos tipos léxicos de verbos (verbos de comunicación, verbos actitudinales, verbos de juicio, verbos de reporte, verbos de cognición, etc.) han sido considerados como prototípicos de los géneros científicos. Tal vez, debido a la prominencia de los estudios léxicos, son escasos en este ámbito los estudios que indagan los rasgos flexivos de los verbos, específicamente, en artículos de investigación. Por su parte, tanto los estudios léxicos como los insuficientes trabajos gramaticales sobre los accidentes del verbo en artículos de investigación, no toman en consideración la sección específica en la que los verbos se utilizan. Si bien existen trabajos de la descripción léxica de los verbos en nuestra lengua, no existen análisis de corte gramatical que incorporen la variable sección. Por otra parte, como en muchos otros campos, la investigación en nuestra lengua, en comparación con la lengua inglesa, es, en general, escasa. En este sentido, y dada la enorme diferencia de la morfología del español con el inglés, los estudios de esta lengua no son directamente comparables con los nuestros.

El objetivo de esta investigación es describir los rasgos flexivos de los verbos de las secciones de artículos de investigación en seis áreas de la ciencia. Conocer los accidentes del verbo en artículos de investigación en español, especificando las secciones en las que se usan, es una ventana que permite conectar esos rasgos formales con las funciones pragmáticas que cumple cada sección en este tipo de textos, el más representativo de los géneros científicos. Esta aproximación proporciona novedosos puntos de conexión de la interfaz triádica que conforman la gramática, la pragmática y el discurso.

Este artículo está organizado de la siguiente forma. En el primer apartado, mostramos los antecedentes de los trabajos en el ámbito específico de esta investigación y especificamos los fundamentos teóricos de las categorías de análisis que utilizamos. En la segunda parte, explicamos detalladamente los procedimientos utilizados en la investigación. En la última sección, presentamos los resultados, su interpretación y las principales conclusiones que podemos obtener de la investigación.

\section{Verbos y artículos De INVESTIGACIÓN}

La descripción del uso de los verbos en artículos de investigación (en adelante, AI) se ha centrado preferentemente en los tipos léxicos de verbos y las funciones que estos cumplen. Una de las funciones prototípicas de los AI es la atribución, es decir, el uso de las ideas de otros investigadores para sustentar teórica y retóricamente la pesquisa propia que se materializa en el AI. Al respecto, son especialmente importantes los denominados verbos de reporte (Thomas y Hawes 1994; Ädel y Garretson 2006). Algunas investigaciones proponen otros tipos léxicos verbales que son de uso común en los AI, detallando las funciones que esta categoría gramatical cumple. FlØttum, Kinn y Dahl (2006) describen el uso de los verbos en AI de economía, lingüística y medicina, asociando su uso a los distintos tipos de roles que asumen los autores 
y que determinan el grado de interacción con el lector. Estos roles que asumen los autores son: el investigador, el escritor, el argumentador y el evaluador. En el estudio de FlØttum, Kinn y Dahl (2006) se concluye que los roles, determinados por el uso de los verbos, son más sensibles a la variable de la disciplina que a la lengua materna del autor. Una dimensión que se considera en ese estudio -aunque de forma parcial- es el hecho de que las formas de los verbos, independientemente del tipo léxico, determinan la interacción con el lector. Por ejemplo, al utilizar el pasado (para explicar los procedimientos), según los autores, se excluye al lector.

Por su parte, Soto, Martínez y Sadowsky (2005) realizan un estudio donde se examina la proporción de verbos y sustantivos en un amplio corpus de textos pertenecientes a las ciencias aplicadas, naturales, sociales y humanidades. Los autores concluyen que la proporción de estas dos categorías permite distinguir textos de especialización distinta, así como también textos de un mismo grado de especialización, pero de disciplinas diferentes. De este modo, entre más especializado el texto, contendrá una menor proporción de verbos en relación a la proporción de sustantivos.

Basándose en el ampliamente aceptado modelo de Thompson y Yiyun (1991), Thomas y Hawes (1994) describen la presencia de tres macrocategorías de verbos en AI de medicina: los "verbos del mundo real", los verbos discursivos y los de cognición. Si bien en ese estudio se analiza la ocurrencia de los verbos en casi todas las partes del AI, los resultados no se muestran desagregados por sección, lo que, entendemos, no permite contar con una descripción más fina del fenómeno. La investigación (Thomas y Hawes 1994) propone una manera específica de vincular la ocurrencia de cierto tipo de verbo con propósitos comunicativos característicos de los AI (hacer referencia general a otros trabajos, explicar procedimientos, persuadir a la audiencia, entre otros). En esta misma línea, Tseng (2011) analiza el uso de los tiempos verbales en 90 resúmenes de artículos de investigación en tres revistas de lingüística aplicada. Usando un modelo de propósitos comunicativos, en el trabajo se concluye que los verbos en presente se utilizan mayoritariamente: para dar los antecedentes de la investigación, para formular el objetivo y para presentar las conclusiones; mientras que para describir los métodos y exponer los resultados, se usa de forma predominante el pasado. Por su parte, en un detallado análisis, Myers (1992) muestra los distintos grados de subjetividad que implica el uso de los verbos de reporte en las oraciones donde se explicita el objetivo de AI de biología y lingüística. La propuesta de Myers es especialmente relevante para la presente investigación, en tanto se asume que el uso de los tiempos verbales en un AI tiene una estrecha relación con la forma en que se presentan los hechos científicos: por ejemplo, cuando se utiliza el presente perfecto en una secuencia como: (1) 'Estos estudios han proporcionado profusa información', los hechos se presentan como incuestionables. Así también, del trabajo de Myers (1992) se advierte que el uso de un tiempo verbal determinado en una sección no supone el mismo valor funcional que cuando se utiliza en otra. Otra evidencia importante que proporciona la descripción de Myers (1992) radica en la diferencia que se muestra entre las disciplinas (la lingüística y la biología). Esto, dado que el uso de ciertas formas verbales reflejan la manera en que cada disciplina conceptualiza la ciencia y, en consecuencia, los hechos científicos. A nuestro parecer, uno de los trabajos más iluminadores en el campo de la descripción de los tiempos verbales en AI es el de Malcom (1987). Esto se debe al carácter explicativo de la 
investigación, en tanto se busca determinar las reglas que determinan el uso de los tiempos verbales en un tipo específico de AI, a saber, los reportes experimentales. El autor propone que, para comprender el uso de los tiempos verbales, se deben considerar dos ejes que configuran el espacio temporal. El primer eje es de carácter referencial y dice relación de forma explícita con el entorno de un experimento: se hace referencia al experimento, a los participantes involucrados, a los procedimientos y resultados del experimento. En este mismo eje se contextualiza el experimento en el ámbito de la investigación pertinente y se discute su relación con el desarrollo de una teoría determinada. El segundo eje es de carácter deíctico y tiene como función ser el medio a través del cual se materializa el acto físico de lectura y escritura del reporte mismo, haciendo referencia al texto mismo, en el que se incluye el material escrito, pero también el material visual que típicamente contienen estos reportes. Otra dimensión que se debe tomar en cuenta, según Malcom (1987), es el significado temporal de los verbos. Para esto, se debe considerar que, si bien los verbos tienen un significado temporal intrínseco, ese significado adquiere valor solo en relación con el momento de la enunciación (time of utterance). Este momento de la enunciación se determina en dos tiempos específicos: a) el momento en que el escritor materializa un enunciado a través de la realización del acto de escritura y b) el momento en que el lector interpreta ese enunciado. A partir de estos ejes y de la determinación del significado de los tiempos verbales, los cuales adquieren valor en relación al momento de la enunciación, el autor (Malcom 1987) propone y comprueba empíricamente tres hipótesis: 1) Las generalizaciones, identificables por el uso de verbos sin un agente "investigador" determinado, se realizan en presente (e.g., 'El futuro de las políticas educativas es incierto'), 2) la referencia a experimentos específicos, identificables por el uso de un agente "investigador" y por la referencia, con una nota al pie, a solo un estudio, se realizan en pasado (e.g., 'Soto (1999) encontró diferencias significativas en el uso de los verbos'), y 3) las referencias a las áreas de la pesquisa, identificables a través de la presencia de un agente investigador y por la referencia, con una nota al pie, a más de un estudio, se realizan en presente perfecto (e.g. 'El estudio de los verbos se ha desarrollado principalmente en el ámbito léxico). Es interesante destacar que en este trabajo, al igual que en otros reseñados (Tseng 2011; Thomas y Hawes 1994), se asocia el uso de ciertos tiempos verbales a la realización de propósitos comunicativos.

Además de estas investigaciones que abordan el uso de los verbos en AI, existen dos trabajos importantes para la presente pesquisa, las cuales, sin embargo, analizan otro género científico, a saber: las tesis. En un estudio de las cláusulas sustantivas reportativas con el complementante que (that), Charles (2006) llega a una conclusión similar a la de Myers (1992). La autora constata en un corpus de tesis de doctorado y magíster, que en esas construcciones la disciplina de la política muestra un uso significativo del presente, en contraste con la ciencia de los materiales, en donde predomina el pasado y el presente perfecto.

En un acucioso trabajo que también analiza tesis (de magíster), en este caso de Biología, Química y Física, Hanania y Akhtar (1985) describen las formas verbales y las funciones retóricas que adoptan en ese género. El interés de esta pesquisa para la presente investigación radica en dos aspectos. En primer lugar, porque el trabajo describe el uso de las formas verbales en secciones determinadas de las tesis y, en segundo lugar, porque la autora asocia el uso de las formas verbales a funciones 
comunicativas específicas. En el estudio se establece que, por ejemplo, independiente de la disciplina, en las introducciones predomina el uso del presente para formular el objetivo de la investigación y realizar generalizaciones. Así también, según las autoras, en la sección de revisión de los antecedentes aumenta el uso del pasado y de tiempos compuestos, especialmente apropiados para reportar las investigaciones previas. En la descripción también se establece que en la sección de métodos, el uso predominante del pasado perfecto y de las formas pasivas se asocia directamente con la descripción de procedimientos y que el uso de los verbos modales y del presente en la discusión, tiene un estrecho vínculo con la evaluación e interpretación de las conclusiones y los resultados. Williams (1996), utilizando un enfoque eminentemente léxico, aporta evidencia respecto del uso de los verbos en dos tipos de AI de medicina, reportes clínicos y experimentales. El autor incluye, como parte de sus resultados, algunas interacciones de los tipos léxicos de verbos con ciertas formas gramaticales específicas. La importancia del trabajo radica en que se muestran diferencias significativas en el uso de los tipos léxicos y las formas verbales entre ambos tipos de géneros (reportes clínicos y experimentales), aun cuando pertenecen a la misma disciplina.

\section{Metodología}

\subsection{Corpus}

El corpus de análisis de esta investigación fue recolectado en el marco del Proyecto FONDECYT 11080097. Se trata del Corpus de Artículos de Investigación en Español (en adelante CaiE), que es representativo de la biblioteca electrónica SciELO-Chile. Para la delimitación del número de casos y su nivel de representatividad, se utilizó una técnica de muestreo aleatorio estratificado con afijación óptima proporcional, de manera que estuvieran representadas todas las disciplinas y las revistas contenidas en la base. Para ello, primero se hizo un estudio exploratorio que tuvo como objetivo conocer qué número de artículos de investigación en español se habían publicado entre los años 2000 y 2008 en la Base SciELO-Chile. En ese trabajo (Sabaj, Matsuda y Fuentes 2010) se generaron criterios funcionales para determinar qué era considerado un artículo de investigación, junto con otros criterios de inclusión y exclusión (idioma y año de publicación) y, de esta forma, se determinó el número de casos totales por cada uno de los estratos (revistas, disciplinas y áreas de la ciencia). Una vez conocido el número de unidades muestrales (en nuestro caso, los artículos) de cada estrato (revistas, disciplinas y áreas de las ciencias), se utilizó la siguiente fórmula para determinar el número de artículos a considerar para contar con un corpus representativo:

Figura 1. Fórmula para el cálculo del tamaño del corpus

$$
n=\frac{N Z_{\frac{\alpha}{2}} s^{2}}{N d^{2}+Z_{\frac{\alpha}{2}} s^{2}}
$$


Donde $n$ representa el tamaño de la muestra a estimar, $N$ es el tamaño de la población, con un nivel de significación alfa del 5\%. Esto implica que nuestra afirmación sobre el tamaño de la muestra tiene un $95 \%$ de probabilidad de ser verdadera. $S^{2}$ representa la estimación de la varianza de la población; $d$ es la precisión o el error del muestreo, en nuestro caso es aproximadamente 0,07688 , el que representa un $7 \%$. Para contar con un corpus representativo, el tamaño $N$ era de 161,6 (número que se aproximó a 162). Luego se utilizó un muestreo aleatorio simple, proporcional a cada estrato, para seleccionar los artículos que conformarían el corpus. Para la clasificación y agrupación de los artículos en disciplinas y áreas de la ciencia, se utilizaron los criterios de la clasificación de las ciencias de la UNESCO. Para más detalles de los procedimientos de recolección, la representatividad y el tipo de muestreo utilizado en el CaiE, véase Sabaj y Matsuda (2010). En la Tabla 1, se muestra la conformación del corpus:

Tabla 1. Conformación del CaiE

\begin{tabular}{|l|c|}
\hline Área de la Ciencia & N \\
\hline Ciencias de la Salud & 75 \\
\hline Ciencias de la Tierra & 11 \\
\hline Ciencias de la Vida & 9 \\
\hline Ciencias Exactas & 9 \\
\hline Ciencias sociales & 45 \\
\hline Humanidades & 13 \\
\hline Totales & $\mathbf{1 6 2}$ \\
\hline
\end{tabular}

\subsection{Modelo para el análisis de los accidentes del verbo}

El análisis de los accidentes del verbo se realizó en forma manual. Para ello, se entrenó a 10 estudiantes. Si bien existen etiquetadores morfológicos y analizadores sintácticos que pudieron haber facilitado la tarea, el tiempo requerido para preparar los textos y la cantidad de errores de estas herramientas, que de igual forma requieren una revisión, así como también el hecho de que las etiquetas de los analizadores morfosintáctico vienen predeterminadas y no admiten modificación, nos llevó a decidirnos por el etiquetaje manual, el cual, si bien es más costoso en términos de tiempo, tiene un nivel de error nulo e incluye las categorías exactas que uno desea observar. El etiquetaje manual, además, tiene la ventaja de que integra en un solo análisis, la caracterización superestructural de los AI y la descripción de los accidentes del verbo.

Para el análisis de los verbos en AI, se usaron las siguientes categorías: el identificador del artículo que permite asociarlo a una disciplina y a un área de la ciencia, la sección analizada, la forma verbal, el lema (infinitivo del verbo), tipo de forma (simple o compuesta), tiempo, persona, número (singular o plural), modo verbal, voz (activa, pasiva con se, o pasiva gramatical), construcciones impersonales y construcciones perifrásticas. Para ver el corpus completo, los identificadores asociados y su clasificación en una disciplina y en un área de la ciencia, véase Sabaj y Matsuda (2010). Para el análisis superestructural que permitió identificar 
la sección analizada, se utilizaron algunos criterios que fueron desarrollados en otra investigación (Sabaj, Matsuda y Fuentes, 2010). De ese trabajo, se concluyó que existen tres tipos de superestructura de los AI del CaiE: a) una superestructura prototípica y rígida, con las categorías típicas de un artículo de investigación científica: Título, Resumen, Introducción, Marco Teórico, Metodología, Resultados, Discusión (o Resultados y Discusión), Conclusión y Referencias Bibliográficas; b) una superestructura más laxa, que corresponde a lo que hemos denominado artículos de investigación o estudio, que no contiene un apartado especial de metodología; y c) una superestructura con categorías temáticas, características de los trabajos de investigación teórica de tipo ensayístico. En el caso de a) se consideró que no siempre las categorías tienen el nombre exacto de la sección, por ejemplo, que en algunas disciplinas, se denomina materiales y métodos a la sección de la metodología, o bien, antecedentes teóricos, a la sección rotulada con el nombre de Marco Teórico. En el caso de b y c, se registraron las categorías superestructurales existentes de forma explícita y se colocaron nombres genéricos para aquellos textos que no presentaran algunas de las categorías superestructurales de forma explícita. Así, por ejemplo, si un texto de carácter teórico tenía una sección Resumen, se registraba tal cual, pero si a continuación no poseía más subtítulos de carácter estructural, lo denominamos introducción, desarrollo y conclusión, de forma que fuera posible realizar una homologación con las partes de un AI con partes prototípicas. En cualquier caso, de ese trabajo se concluyó que la mayoría (un 70\%) de los AI del CaiE tenían secciones claramente identificables. En la sección de resultados pueden revisarse las secciones consideradas en esta investigación, así como las posibles combinaciones (Resultados y Discusión, Discusión y Conclusión) que emergieron del análisis.

La forma verbal correspondía al extracto verbal tal cual como aparecía en el texto. Se incluyó en esta categoría la partícula se para identificar si se trataba de una forma impersonal o una estructura pasiva. El tipo de forma se clasificó en simples y compuestas. En las compuestas se consideraron las estructuras con verbo auxiliar y también las estructuras perifrásticas. Cada forma se lematizó con el infinitivo, de manera que fuese posible tener un listado del vocabulario verbal de cada artículo. Si bien la lematización es un procedimiento muy fácil para el caso de las formas simples, en las estructuras perifrásticas no es del todo evidente. Para el análisis del modo, la persona y el número se emplearon las categorías típicas que aparecen en cualquier gramática. Para el análisis de los tiempos se utilizaron las siguientes etiquetas: presente, presente perfecto, pasado simple, pasado imperfecto, pasado pluscuamperfecto, futuro, futuro perfecto, condicional, condicional perfecto. En el análisis de la voz, se diferenciaron las estructuras activas, las pasivas con se y las pasivas gramaticales (verbo ser en pasado + participio). En una columna aparte, se registró además si la forma analizada era impersonal y si correspondía a una perífrasis, dato que permite diferenciar las formas compuestas propiamente tales (verbo auxiliar + participio) de las formas compuestas perifrásticas.

Para todos los casos dudosos, se les pidió a los etiquetadores que dejaran el análisis pendiente. Todos esos casos fueron resueltos de forma conjunta, una vez que se consolidaron en una misma base de datos los análisis particulares de cada asistente. Para facilitar el posterior análisis estadístico, los datos se registraron en una hoja de cálculo, tal como se muestra en el ejemplo a continuación (por razones de espacio hemos omitido los primeros tres campos, a saber, ID, el área de la Ciencia y la disciplina): 
Tabla 2. Ejemplo del análisis

\begin{tabular}{|c|c|c|c|c|c|c|c|c|c|c|c|c|}
\hline S & F & L & TF & T & P & N & M & Act. & $\begin{array}{c}\text { Pas. } \\
\text { Con Se }\end{array}$ & Pas. & I & PV \\
\hline Resumen & mostraron & Mostrar & simple & $\begin{array}{c}\text { Pasado } \\
\text { simple }\end{array}$ & tercera & plural & Indicativo & 1 & 0 & 0 & 0 & 0 \\
\hline Resumen & sostienen & sostener & Simple & Presente & tercera & plural & Indicativo & 1 & 0 & 0 & 0 & 0 \\
\hline
\end{tabular}

Leyenda: S: Sección; f: Forma verbal; TF: Tipo de forma; L: Lema; T: Tiempo; P: Persona; N: Número; M: Modo; Act.: Activa; Pas. con Se: Formas pasivas con se; Pas. : Forma pasiva gramatical; I: Impersonal; PV: Perífrasis verbal.

\section{Resultados y DiscusióN}

Se identificaron y analizaron 42.086 formas verbales en los 162 artículos que componen el CaiE, correspondientes a 7.093 tipos de lemas distintos. Los lemas dan cuenta de la cantidad de vocabulario de los textos, mientras que las formas corresponden a todos los casos encontrados.

Para efectos de los resultados que se exponen a continuación, la muestra está constituida por los 42.086 verbos encontrados, que constituyen el 100\% respecto del cual se obtendrán los porcentajes. Por razones de espacio, no se muestran todos los resultados con el mismo nivel de detalle. ${ }^{1}$ En la primera parte, se expondrán de forma global algunos patrones generales según las áreas de la ciencia. En un segundo punto, se mostrarán en detalle los porcentajes de cada grupo de variables por las secciones del artículo de investigación.

\subsection{Resultados generales por área de la ciencia}

La tercera persona singular es la que muestra el mayor uso en todas las áreas de la ciencia con un promedio de $61,7 \%$, seguida por la tercera persona plural con un $35 \%$. La primera persona singular y la segunda persona en singular y en plural no superan en ninguna área de la ciencia el 1\% de uso. Si bien el uso de la primera persona plural es bajo en todas las áreas de la ciencia, en las Humanidades tiene su valor máximo con un $6,95 \%$, seguida por las Ciencias Sociales con un 4,18\%. El mayor uso de la tercera persona singular puede asociarse a su relación con el discurso expositivo: es esta persona la que se utiliza para hacer aseveraciones, describir fenómenos o teorías; en síntesis, es la persona que se utiliza para hablar del mundo.

El modo más frecuente en todas las áreas de la ciencia es el indicativo con un 95, $1 \%$ promedio en el corpus. El modo subjuntivo presenta un rango de aparición bajo que va del 7\% máximo (en el caso de las Ciencias Exactas) a un 0,8\% mínimo (en las Ciencias de la Tierra). En promedio, el subjuntivo aparece en un 4,9\% de las 42.086 formas identificadas. Las Ciencias Sociales presentan un 6,8\% de los casos en modo subjuntivo y un $5,2 \%$ en las Humanidades. Es interesante destacar que sean las Ciencias Exactas las con mayor uso de este modo, el cual está asociado

\footnotetext{
Se decidió mostrar los resultados más detallados por sección que por área de la ciencia. Los autores pueden proveer a los lectores interesados, el detalle de todos los resultados.
} 
a la expresión de subjetividad. Según este criterio, las Ciencias Exactas serían más subjetivas que las Ciencias de la Tierra, en tanto es el área con menor uso de este modo. Sin embargo tal interpretación no es pertinente, dado que este modo se utiliza también para otras funciones, aparte de expresar subjetividad, por ejemplo, interpretar datos. Solo existen 3 casos de uso del modo imperativo en el corpus, los cuales aparecen en el área de las Ciencias Sociales (se trata de un texto citado, específicamente la transcripción de una conversación) y representan solo un $0,015 \%$ de los 42.086 verbos analizados.

Las formas simples son predominantes en todas las áreas de la ciencia, y representan un $80,3 \%$ de todos los verbos del corpus. Las formas compuestas aparecen solo en un $19,7 \%$ de las 42.086 formas verbales analizadas. Las formas compuestas tienen el uso más bajo en las Ciencias de la Tierra. En todo el resto de las áreas (Ciencias de la Salud, Ciencias de la Vida, Ciencias Exactas, Ciencias Sociales y Humanidades), las formas compuestas tienen un comportamiento muy homogéneo, que va desde un $20,4 \%$ en las Ciencias Sociales, a un $22,7 \%$ en las Ciencias Exactas.

El tiempo más frecuente en todas las áreas de la ciencia es el presente, con un promedio de $62,7 \%$. En el caso de las formas simples, la mayoría de las áreas de la ciencia muestran el siguiente patrón en el uso de los tiempos verbales: Presente> Pasado Simple $>$ Pasado imperfecto $>$ Condicional $>$ Futuro. El mayor uso del pasado imperfecto aparece en las Ciencias Sociales (13,6\%) y en las Ciencias de la Tierra (11\%).

La predominancia del modo indicativo, el tiempo presente y la tercera persona singular en todas las áreas de la ciencia puede explicarse, en parte, porque se trata de elementos no marcados. Tal como demuestra Croft (2003), el uso de los elementos no marcados es siempre, en todas las lenguas, mayor que los elementos marcados. Su mayor utilización radicaría también en el hecho de que estos elementos tienen un mayor potencial funcional; como lo no-marcado es más general, se puede utilizar para más efectos. Así, por ejemplo, el presente tiene un potencial de significados mucho mayor (presente histórico, futuro deíctico, habitual) que todos los otros tiempos verbales. Si bien el uso extensivo del indicativo puede explicarse por el mismo fenómeno, creemos que el tinte de objetivismo con el que se maquilla el discurso científico también juega un papel en este caso. En general, la ciencia (al menos en este género específico, el artículo de investigación), no es un lugar para especular, expresar duda o subjetividad, todas funciones comúnmente asociadas al modo subjuntivo.

En una mirada general, los accidentes del verbo no muestran mayor variación en las distintas áreas de la ciencia. Esto puede deberse a una suerte de primacía del género por sobre los cambios atribuibles a las diferencias conceptuales entre las áreas de la ciencia. Aunque no es el propósito de este trabajo, es muy probable que en cualquier género los elementos no marcados sean los más frecuentes, pero que su proporción en relación con los elementos marcados sea diferente. Por ejemplo, si se analiza un corpus de textos narrativos, es probable que el presente se utilice más que el pasado y que el indicativo sea más usado que el subjuntivo. Sin embargo, al comparar dos géneros, por ejemplo, artículos de investigación y textos literarios, la proporción del pasado y del subjuntivo en estos últimos será mayor que en los primeros. En cualquier caso, considerando la idea de Croft (2003), en ambos géneros primarán siempre los elementos no marcados. Esta hipótesis debería poder confirmarse con estudios ulteriores. 


\subsection{Resultados por sección}

En la Tabla 3, se exponen los resultados relativos a la distribución porcentual de las personas gramaticales en las secciones de los artículos del corpus:

Tabla 3. Porcentaje de uso de las personas gramaticales por sección

\begin{tabular}{|l|c|c|c|c|c|c|c|}
\hline & \multicolumn{2}{|c|}{ Primera } & \multicolumn{2}{c|}{ Segunda } & \multicolumn{2}{c|}{ Tercera } & \\
\hline Sección & Singular & Plural & Singular & Plural & Singular & Plural & Totales \\
\hline Resumen & 0,09 & 2,47 & 0,00 & 0,00 & 62,52 & 34,83 & 100 \\
\hline Introducción & 0,51 & 2,91 & 0,00 & 0,04 & 61,01 & 35,53 & 100 \\
\hline Marco Teórico & 0,05 & 6,56 & 0,00 & 0,02 & 65,14 & 28,23 & 100 \\
\hline Desarrollo & 0,54 & 3,34 & 0,00 & 0,04 & 68,05 & 28,03 & 100 \\
\hline Metodología & 0,27 & 2,05 & 0,02 & 0,00 & 64,07 & 33,59 & 100 \\
\hline Resultados & 0,12 & 1,84 & 0,00 & 0,00 & 67,76 & 30,27 & 100 \\
\hline Resultados y discusión & 0,60 & 3,74 & 0,00 & 0,00 & 55,15 & 40,51 & 100 \\
\hline Discusión & 0,34 & 4,71 & 0,00 & 0,02 & 64,94 & 29,98 & 100 \\
\hline Discusión y conclusión & 0,00 & 4,38 & 0,00 & 0,00 & 62,04 & 33,58 & 100 \\
\hline Conclusión & 0,27 & 3,65 & 0,00 & 0,00 & 67,17 & 28,91 & 100 \\
\hline Agradecimientos & 3,33 & 15,00 & 0,00 & 0,00 & 38,33 & 43,33 & 100 \\
\hline
\end{tabular}

En el caso de la primera persona, las formas plurales son en todas las secciones más frecuentes que las formas singulares. Si bien el número de autores por artículo no fue un criterio considerado en la recolección del corpus, el uso de las formas plurales en todas las secciones puede deberse al intento de objetivizar el discurso a través del denominado nosotros de modestia, aunque esto no aplica en aquellos artículos con más de un solo autor. La diferencia entre la forma plural y la singular está más marcada en la sección del Marco Teórico (0,05 \% vs. 6,56 \%) y en los Agradecimientos (3,33\% vs. 15,00\%). En el primer caso, la primera persona plural se utilizaría para adoptar una posición respecto de las teorías (e.g., 'Concordamos con Soto (1999) en que las teorías de la mente...') o para apropiarse de los conceptos que aparecen típicamente en esta sección (e.g., 'Entendemos la noción de mente como un dispositivo...'). En los Agradecimientos, el uso del plural puede explicarse porque el sujeto que agradece, independientemente de si se trata de uno o varios autores, adopta por convención esta forma plural.

El uso de la segunda persona es casi nulo en todas las secciones. Revisando los casos en detalle, se puede establecer que se trata de citas textuales a entrevistas. Este resultado es esperable, dado el carácter del género analizado, en el cual la identificación del lector a través del uso de la segunda persona es inexistente.

Tal como lo mencionamos en la descripción de los resultados generales por área de la ciencia, la tercera persona singular es la forma más empleada en la mayoría 
de las secciones de los artículos de investigación, a excepción de la sección de los Agradecimientos. Sin considerar esta sección, el promedio del uso de la tercera persona singular alcanza en promedio un $67 \%$ versus un $33 \%$ de uso de las formas plurales. El mayor empleo de la tercera persona singular por sobre la plural puede deberse a la mayor cantidad de funciones que se pueden realizar con esta forma. Al analizar la tercera persona, la diferencia entre las formas plurales y las singulares se estrecha en los artículos que contienen una sección conjunta de Resultados y discusión (55,15\% vs. $40,51 \%$ ). Si bien la forma singular sigue primando, la mayor aparición de las formas plurales puede deberse a que, por definición, en ningún artículo se muestra un solo resultado, de forma que al referirse a ellos, necesariamente se debe utilizar el plural.

En la Tabla 4, se exponen los resultados del uso de los modos en las secciones de los artículos de investigación.

Tabla 4. Porcentaje de uso de los modos del verbo en las secciones de los artículos de investigación

\begin{tabular}{|l|c|c|c|c|}
\hline Sección & Indicativo & Subjuntivo & Imperativo & Totales \\
\hline Resumen & 98,08 & 1,92 & 0,00 & 100 \\
\hline Introducción & 97,11 & 2,89 & 0,00 & 100 \\
\hline Marco Teórico & 94,01 & 5,97 & 0,02 & 100 \\
\hline Desarrollo & 94,79 & 5,21 & 0,00 & 100 \\
\hline Metodología & 97,28 & 2,72 & 0,00 & 100 \\
\hline Resultados & 94,40 & 5,60 & 0,00 & 100 \\
\hline Resultados y discusión & 96,75 & 3,25 & 0,00 & 100 \\
\hline Discusión & 93,50 & 6,48 & 0,02 & 100 \\
\hline Discusión y conclusión & 100,00 & 0,00 & 0,00 & 100 \\
\hline Conclusión & 95,17 & 4,83 & 0,00 & 100 \\
\hline Agradecimientos & 100,00 & 0,00 & 0,00 & 100 \\
\hline
\end{tabular}

Tal como sucede al comparar las áreas de la ciencia, el modo que prima en todas las secciones es el indicativo. El modo subjuntivo tiene un mayor uso en las secciones Discusión, Marco Teórico, Desarrollo, Resultados, Resultados y Discusión. El uso del subjuntivo en estas secciones se puede explicar por la expresión de la subjetividad; en el Marco Teórico y en el Desarrollo, para posicionarse con respecto a conceptos y teorías. En las otras secciones (Discusión, Resultados, Resultados y Discusión), el uso del subjuntivo se justifica con relación a las funciones típicas que aparecen en dichas secciones, a saber, discutir e interpretar los resultados propios, contrastarlos con los de otras investigaciones o buscar las posibles causas de la obtención de esos resultados. Destaca el nulo uso del subjuntivo en aquellos artículos que combinan la Discusión y la Conclusión, como también el bajo uso de este modo en el Resumen y la Metodología. En el Resumen y la Metodología, el bajo uso del subjuntivo puede asociarse a la pretensión de objetividad que suele asociarse a estas secciones. 
En la Tabla 5, se muestra el porcentaje de uso de los tiempos verbales en el corpus analizado:

Tabla 5. Uso de los tiempos verbales por sección

\begin{tabular}{|c|c|c|c|c|c|c|c|c|c|c|}
\hline \multirow[b]{2}{*}{ Sección } & \multicolumn{5}{|c|}{ Formas simples } & \multicolumn{4}{|c|}{ Formas compuestas } & \multirow[b]{2}{*}{ Totales } \\
\hline & Pres & Pimp & Psimp & Fut & Cond & Preperf & Pрсиат & Futperf & Conperf & \\
\hline Resumen & 60,24 & 3,11 & 29,98 & 1,65 & 1,37 & 2,74 & 0,82 & 0,09 & 0,00 & 100,00 \\
\hline Introducción & 68,00 & 4,39 & 13,24 & 1,88 & 3,34 & 8,49 & 0,56 & 0,00 & 0,11 & 100,00 \\
\hline Marco Teórico & 62,95 & 10,52 & 13,18 & 2,85 & 4,65 & 4,48 & 0,57 & 0,00 & 0,80 & 100,00 \\
\hline Desarrollo & 60,32 & 9,26 & 19,35 & 1,79 & 2,63 & 5,21 & 1,25 & 0,04 & 0,15 & 100,00 \\
\hline Metodología & 49,70 & 5,31 & 39,51 & 1,12 & 1,21 & 2,47 & 0,63 & 0,00 & 0,04 & 100,00 \\
\hline Resultados & 59,17 & 9,97 & 21,40 & 2,16 & 3,78 & 2,72 & 0,64 & 0,07 & 0,09 & 100,00 \\
\hline $\begin{array}{l}\text { Resultados y } \\
\text { discusión }\end{array}$ & 73,48 & 1,39 & 15,85 & 0,78 & 3,50 & 4,40 & 0,06 & 0,00 & 0,54 & 100,00 \\
\hline Discusión & 54,67 & 14,97 & 16,10 & 1,98 & 5,84 & 4,64 & 1,07 & 0,03 & 0,70 & 100,00 \\
\hline $\begin{array}{l}\text { Discusión y } \\
\text { conclusión }\end{array}$ & 70,07 & 1,46 & 11,68 & 1,46 & 13,87 & 1,46 & 0,00 & 0,00 & 0,00 & 100,00 \\
\hline Conclusión & 66,72 & 11,93 & 10,94 & 1,82 & 3,46 & 4,60 & 0,38 & 0,00 & 0,15 & 100,00 \\
\hline Agradecimientos & 56,67 & 0,00 & 35,00 & 0,00 & 1,67 & 5,00 & 0,00 & 0,00 & 1,67 & 100,00 \\
\hline
\end{tabular}

Leyenda: Pres $=$ Presente; Pimp $=$ Pasado imperfecto; Psimp $=$ Pasado simple; Fut $=$ Futuro; Cond $=$ Condicional; Preperf $=$ Presente perfecto; Ppcuam=Pasado pluscuamperfecto; Futperf $=$ Futuro perfecto; Conperf $=$ Condicional perfecto

Al comparar las tablas anteriores con lo que se muestra en la Tabla 5, se puede establecer que el accidente del verbo con mayor variación en las secciones de los artículos de investigación es el tiempo verbal. Si bien persiste la predominancia del elemento no marcado (el presente) en todas las secciones, la variación es mayor que en todos los otros accidentes analizados por sección, que, como hemos visto, tienen un comportamiento bastante homogéneo. En general, en el caso de las formas simples, el patrón de uso de los tiempos verbales sigue para todas las secciones el siguiente esquema (el mismo que sigue en las áreas de la ciencia): Presente $>$ Pasado simple $>$ Pasado imperfecto $>$ Condicional $>$ Futuro.

Para explicar el uso de los tiempos verbales en las secciones en los artículos de investigación, es de utilidad la distinción entre dos ejes temporales que realiza Malcom (1987), a saber, el eje referencial y el eje deíctico. Tal como lo hemos descrito, el primero hace referencia al experimento, a sus participantes y a los procedimientos. En el segundo caso (el eje deíctico), dice relación con el acto físico de la lectura y escritura del texto, así como también a las referencias al texto mismo y al material visual que típicamente aparece en este tipo de textos. Una forma más simple de entender estos ejes es la siguiente. Todo proceso investigativo puede dividirse en dos partes: la realización de la investigación propiamente tal y el reporte de dicha investigación en la forma de un escrito. El reporte es necesariamente posterior a la realización de 
la investigación. Estos dos momentos serían equivalentes al eje referencial y al eje deíctico que postula Malcom (1987). Las secciones de los artículos de investigación, en este sentido, combinan la presentación de estos momentos, es decir, el proceso de investigación realizado y el texto en que se presenta este proceso. Así, por ejemplo, el mayor uso del pasado simple en la sección Metodología $(39,51 \%)$ puede explicarse ya que es la sección donde típicamente se hace referencia al momento relativo al proceso de investigación. En el Resumen, en tanto, para todo lo relativo a la presentación del texto mismo se usa el presente, pero también se usa el pasado, puesto que en esa sección también se hace referencia al proceso de investigación, por ejemplo, cuando en el Resumen se muestra la metodología. Lo mismo puede sostenerse en relación a las secciones de Resultados y Resultados y Discusión. En estas secciones, el autor estratégicamente puede presentar sus resultados relacionados al momento del proceso de investigación (e.g. 'El índice de correlación obtenido fue de 0,75') o bien, en presente (en el momento del reporte del texto), haciendo coincidir la presentación de esos resultados con el tiempo de la lectura del artículo (e.g. 'Los resultados muestran que los sujetos del grupo experimental tienen mejor comprensión de lectura que los sujetos del grupo control'). Se podría interpretar que en el segundo caso, al usar el presente, de una u otra forma, el autor hace partícipe al lector en la presentación de esos resultados: "esto muestra algo que tú, lector, puedes observar, en el momento mismo que yo, autor, te lo presento".

La Introducción es una sección donde, al igual que en el resumen, se combina la presentación del proceso de investigación con la presentación del texto mismo. Eso podría explicar el uso del pasado simple $(29,98 \%)$ y del presente $(60,24 \%)$ en esa sección. El mayor uso del presente perfecto $(8,49 \%)$ en la Introducción puede explicarse por la función de posicionar el trabajo, fundiendo los dos momentos, i.e., el proceso de investigación con el reporte del mismo a través del texto (e.g 'Distintos trabajos han descrito las características del discurso argumentativo.').

El uso del pasado imperfecto y del condicional aparece asociado a aquellas secciones donde se desarrolla la teoría (Marco Teórico, Desarrollo) y a aquellas donde se presentan, se discuten o interpretan los resultados (Resultados, Discusión, Resultados y Discusión, Conclusión).

Tal como sucede al analizar las áreas de la ciencia, en el caso de las secciones, las formas compuestas tienen un uso muy bajo. Entre ellas, se destaca el uso del presente perfecto, el cual, como hemos sostenido, puede cumplir funciones diferenciadas en cada una de las secciones.

\section{Conclusiones}

En este artículo, hemos descrito el uso de los accidentes de 42.086 formas verbales en 162 artículos de investigación. Los resultados expuestos permiten establecer las siguientes conclusiones.

Los accidentes del verbo más frecuentes son los elementos no marcados, específicamente, el tiempo presente, el modo indicativo y la tercera persona singular. Desde un punto de vista teórico, esto se debe a que estos elementos son más polifuncionales; por ejemplo, el presente puede adquirir muchos más valores que el pasado. Por otra parte, hemos establecido que en el uso de los verbos existen ciertos patrones que no son afectados por las áreas del conocimiento de las cuales se obtuvieron los artículos analizados. 
Al analizar el uso de los verbos por sección, el único accidente que varía es el tiempo verbal, aunque sigue siendo el elemento no marcado el más frecuente, a saber, el tiempo presente. El uso de ciertos tiempos verbales en determinadas secciones de los artículos de investigación puede explicarse por un asunto estratégico. El autor de un artículo debe, por un lado, remitirse al proceso de investigación que llevó a cabo y, por otro, presentar retóricamente el escrito que reporta ese proceso. Así por ejemplo, en aquellas secciones en las que típicamente se hace referencia al proceso de investigación (la metodología), se utilizará más el tiempo pasado, mientras que en aquellas donde se deben combinar ambos aspectos, la proporción entre el uso del presente y el pasado será más estrecha.

Los resultados de esta investigación pueden ser comparados con datos similares obtenidos en otros tipos de textos, siendo esta la proyección natural de la presente pesquisa.

Los hallazgos de esta investigación pueden ser de utilidad para aquellos interesados en la descripción del discurso científico y en la didáctica de la escritura científica.

\section{OBRAS CITADAS}

Ädel, Annelie y Garretson, Gregory. 2006. "Citation practices across the disciplines: The case of proficient student writing", 271-280. Conferencia en AELFE 5, Zaragoza, España, 14-16 September 2006.

Charles, Maggie. 2006. "Phraseological patterns in reporting clauses used in citation: A corpusbased study of theses in two disciplines". English for Specific Purposes 25, 3: 310-331.

Croft, William. 2003. Typology and Universals. Cambridge: Cambridge University Press.

FlØttum, Kjersti; Kinn, Torodd y Dahl, Trine. 2006. "We now report on ..." versus " Let us now see how ...': Author roles and interaction with readers in research articles" [en línea]. Disponible en http://www.hf.uib.no/forskerskole/FlottumKinnDahl.pdf

Hanania, Edith y Akhtar, Karima. 1985. "Verb Form and Rhetorical Function in Science Writing". The ESP Journal 4: 29-58.

Malcom, Lois. 1987. "What rules govern tense usage in scientific articles?" English for Specific Purposes 6, 1: 31-43.

Myers, Greg. 1992. "In this paper we report ...: Speech acts and scientific facts". Journal of Pragmatics 17, 4: 295-313.

Sabaj, Omar y Matsuda, Ken. 2010. Informe CaiE [en línea]. Disponible en: http://omarsabaj. wordpress.com/anexos-investigaciones/

Sabaj, Omar; Matsuda, Ken y Fuentes, Miguel. 2010. "Un modelo para la homogeneización de las clases textuales de la biblioteca electrónica SciELO-Chile: La variabilidad del artículo de investigación en diversas disciplinas". Información Tecnológica 21, 6: 133-148.

Soto, Guillermo; Martínez, Ricardo \& Sadowsky, Scott (2005). "Verbos y sustantivos en textos científicos. Análisis de variación en un corpus de textos de ciencias aplicadas, naturales, sociales y humanidades". Philologia Hispalensis 19: 169-187.

Thomas, Sarah y Hawes, Thomas. 1994. "Reporting verbs in medical journal articles". English for Specific Purposes 13, 2: 29-148.

Thompson, Geoff y Yiyun, Ye. 1991. "Evaluation in the Reporting Verbs Used in Academic Papers". Applied Linguistics 12, 4: 365-82.

Tseng, Fan-ping. 2011. "Analyses of move structure and verb tense of research article abstracts in applied linguistics". International Journal of English Linguistics 1, 2: 27-39.

Williams, Ian. 1996. "A Contextual Study of Lexical Verbs in Two Types of Medical Research Report: Clinical and Experimental”. English for Specific Purposes 15, 3: 175-197. 